\title{
Cadmium in marine invertebrates and arctic cod in the Canadian Arctic. Distribution and ecological implications
}

\author{
C. R. Macdonald ${ }^{*}$, J. B. Sprague \\ Department of Zoology, University of Guelph, Guelph, Ontario, Canada N1G 2W1
}

\begin{abstract}
Seals and other marine mammals in the North American Arctic have high concentrations of cadmium in the kidney, particularly in relation to northern European seals. We measured cadmium in other organisms to determine whether the metal was generally elevated in Canadian arctic marine ecosystems. Samples of dominant organisms were collected from the epontic (under-ice) community in the central Canadian Arctic in 1983 and 1984, and in the pelagic community in the eastern Canadian Arctic in 1982 and 1983. Cd concentrations in invertebrates ranged from $0.17 \mathrm{\mu g} \mathrm{g}^{-1}$ in Mysis litoralis to consistently high concentrations of 14 to $16 \mu \mathrm{g} \mathrm{g}^{-1}$ in hyperiid amphipods. These were equivalent to, or lower than, the values in corresponding organisms outside the Arctic, including hyperiid amphipods which have similarly high levels in the north Pacific Ocean. With the exception of 1 species of amphipod (Gammarus setosus), there were no significant differences $(p>0.05)$ between sampling sites or years. Arctic cod Boreogadus saida, an important food item for most arctic vertebrates, ranked among the lowest of the Canadian arctic organisms in Cd concentration $\left(0.40 \mu \mathrm{g} \mathrm{g}^{-1}\right)$, but was appreciably higher than fish from northern Europe (Baltic Sea). Except for arctic cod, there was no evidence of elevated Cd concentrations unique to the marine community of the Arctic. High Cd levels in arctic marine mammals should not be taken as evidence of general ecosystem contamination. However, the high levels in hyperiid amphipods, notably Parathemisto libellula, take on a particular significance because the amphipods are prominent in the diet of many arctic marine vertebrates.
\end{abstract}

\section{INTRODUCTION}

High levels of cadmium in the liver and kidney of marine mammals have been reported for the North American Arctic and Greenland, areas not known to be subjected to high inputs of the metal (Rahn 1981). Mean kidney concentrations are $267 \mu \mathrm{g} \mathrm{g}^{-1}$ dry weight (SD 97.7) in northern fur seals Callorhinus ursinus in Alaska (Goldblatt \& Anthony 1983) and $150^{\dagger} \mu \mathrm{g} \mathrm{g}^{-1}$ (range $36^{\dagger}$ to $585^{\dagger}$ ) in ringed seals Phoca hispida from western Greenland (Johansen et al. 1980). In Greenland seals, the maximum value approaches the critical concentration of $800^{\dagger} \mu \mathrm{g} \mathrm{g}^{-1}$ in the kidney cortex at

\footnotetext{
- Present address: Environmental and Resource Studies, Trent University, Peterborough, Ontario, Canada K9J 7B8

$\dagger$ Tissue concentrations marked with a cross were originally reported in terms of wet weight but have been multiplied by 4.0 as a general conversion factor to dry weight. The factor is used here for conversions of concentrations in both the whole body and in specific organs, to simplify the reader's comparisons within this paper
}

which kidney dysfunction is expected to occur in humans (Friberg et al. 1974), assuming a conservative cortex/whole kidney concentration ratio of 1.25 (Cherry 1981). These tissue levels are very high compared to those for seals in northern European waters; ringed seals in the Gulf of Finland have a mean kidney concentration of only $4.8^{\dagger} \mu \mathrm{g} \mathrm{Cd} \mathrm{g}{ }^{-1}$ in kidney (range $0.16^{\dagger}$ to $16^{\dagger}$ ) although they contain relatively high levels of contaminants such as mercury $\left(48^{\dagger} \mu \mathrm{g} \mathrm{g}^{-1}\right)$ in liver and PCBs $\left(300^{\dagger} \mu \mathrm{g} \mathrm{g}^{-1}\right)$ and total DDT $\left(300^{\dagger} \mu \mathrm{g}\right.$ $\mathrm{g}^{-1}$ ) in blubber (Pertilla et al. 1986).

There are few data to provide a background for explaining the high $\mathrm{Cd}$ concentrations in the North American arctic mammals. Bohn \& McElroy (1976) reported whole-body and liver concentrations of $\mathrm{Cd}$ in arctic cod Boreogadus saida from the Canadian eastern Arctic, and also values for copepods that were well within the range reported for copepods from uncontaminated areas outside the Arctic (Table 5). Although some studies have reported slightly higher concentrations of dissolved $\mathrm{Cd}$ in surface waters of the Arctic 


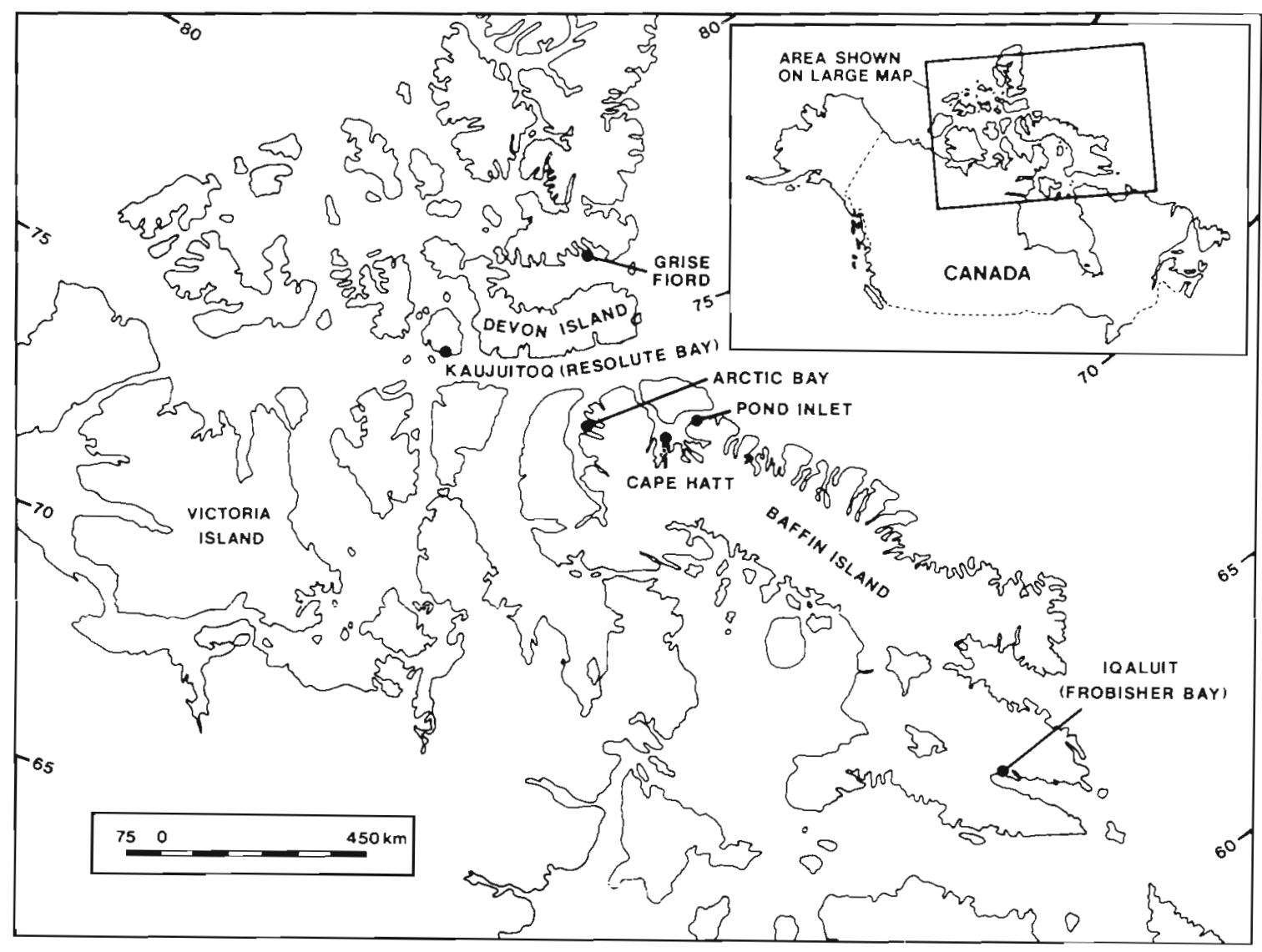

Fig. 1. Study areas in the Northwest Territories of Canada

than would be expected from temperate regions (Moore 1981, Kremling 1985) there has been no thorough evaluation of Cd concentrations in the lower parts of the food web and no apparent reason for high tissue levels in arctic seals and some other mammals.

The present study was undertaken to document $\mathrm{Cd}$ concentrations in organisms of the arctic marine community and to relate the levels observed in the food web to those in the marine mammals. Our hypothesis was that $\mathrm{Cd}$ concentrations in the organisms would reflect, in general, the elevated concentration of $\mathrm{Cd}$ in arctic waters noted in other studies, and that the seals in the Canadian Arctic would show proportional elevations similar to other organisms. The initial objective was therefore to collect representative organisms from all trophic levels of Canadian arctic marine ecosystems and measure their $\mathrm{Cd}$ content. Ultimately, it is intended to use the measured concentrations to test whether the differences in tissue concentrations between ringed seals in Europe and Canada result from differences in $\mathrm{Cd}$ content of the respective diets. This paper reports

- Place names attempt to reflect recent changes from English to Inuktitut the results of the collection and analysis of organisms from the spring ice community near Kaujuitoq " (Cornwallis Island) and the open water community of northern Baffin Island, in the central and eastern Canadian Arctic, and examines their significance with respect to marine mammals.

\section{MATERIALS AND METHODS}

Collection of organisms. Pelagic, benthic and intertidal organisms were collected in the waters surrounding Cape Hatt, on northern Baffin Island, N.W.T. (Fig. 1) in the last $2 \mathrm{wk}$ of August 1982 and 1983. Full descriptions of aquatic species in the area are given in Sekerak et al. (unpubl., cited in Bradstreet 1982). All sampling was conducted from the camp of the Baffin Island Oilspill Program (BIOS) at Cape Hatt, hence the present study uses the names for individual bays (i.e. Z-lagoon, Bay 12) used by that project (for description of project see Arctic, Vol. 40, Suppl. 1, 1987).

In 1982, pelagic zooplankton were collected with either a $0.25 \mathrm{~m}^{2}$ conical net with a PVC cod bucket, or with a $0.10 \mathrm{~m}$ diameter Miller sampler, towed from a 
$4.3 \mathrm{~m}$ Zodiac inflatable boat at approximately 2 to 3 knots. To reduce contamination, nets were constructed of nylon mesh, canvas and PVC or fiberglass. The hoop of the conical nets was made of $9.5 \mathrm{~mm}$ tubular stainless steel while the tow ropes and bridles were $8 \mathrm{~mm}$ diameter polypropylene. Mesh sizes were 64, 273, 505, and $1050 \mu \mathrm{m}$. In 1983, all pelagic invertebrates were collected using the same conical nets equipped with a $3.6 \mathrm{~kg}$ hydrodynamic depressor. This sampled at greater depths (20 to $25 \mathrm{~m}$ ), and collected more specimens, especially of copepods, pelagic amphipods, and chaetognaths.

Intertidal amphipods were collected during low tide using hand-held dip nets. Scavenging benthic amphipods were collected with a minnow trap covered with $273 \mu \mathrm{m}$ mesh, baited with the head of fresh arctic char Salvelinus alpinus and retrieved from 3 to $5 \mathrm{~m}$ depth after 18 to $36 \mathrm{~h}$, or in one instance, by a dip net as they consumed a fish in a gill net. There was no indication of contamination using the metal minnow trap since there was no significant difference $(p>0.05)$ in the $\mathrm{Cd}$ concentrations of amphipods collected by the 2 methods.

Frozen samples of the pelagic amphipod Parathemisto libellula, and arctic cod, were obtained from the gut of harp seals taken at Grise Fiord, N.W.T., courtesy of D. Lavigne and D. Murie, University of Guelph. Freeze-dried P. libellula, from Iqualuit (formerly Frobisher Bay), N.W.T. were provided by J. Fife, Arctic Biological Station, Ste-Anne de Bellevue, P. Q.

Pelagic invertebrates were also collected earlier in the season, under the ice, in June 1983 and 1984 in Barrow Strait, immediately south of Kaujuitoq (formerly Resolute Bay), N.W.T. In both years the conical nets described above were used through holes or leads in the ice, and remained in the current under the ice for 10 to $15 \mathrm{~min}$ at depths of 10 to $40 \mathrm{~m}$.

Epontic algae were collected in June 1983 by taking cores of $23 \mathrm{~cm}$ diameter through the ice with a stainless steel corer, melting the lower portion, and passing the water through a pre-washed, dried, and pre-weighed $0.45 \mu \mathrm{m}$ Millipore filter. In 1984, samples of the epontic community were collected into a plastic tank by a suction apparatus held against the bottom of the ice by a diver. The water was passed through $64 \mu \mathrm{m}$ nylon mesh and then through a $0.45 \mu \mathrm{m}$ Millipore filter. The material retained by the $64 \mu \mathrm{m}$ mesh (primarily harpacticoid copepods) and the filters were then frozen. Samples from both years contained a wide range of organisms including algae, calanoid and harpacticoid copepods and small amphipods, hence they will be called 'algal community' or 'epontic community' samples rather than algal samples. Large under-ice amphipods (Gammarus setosus) were collected in 1984 by divers using dip nets.
Immediately after collection, the organisms were sorted to species and, if possible, to size-class, partially dried by removal of surface water, and stored in acidwashed Petri dishes. Individual organisms were arranged in the dishes so that they did not come in contact with each other, allowing subsequent analysis of the $\mathrm{Cd}$ content of each organism. Copepods, chaetognaths, mysids, medusae and pteropods were grouped in cleaned plastic vials and subsamples taken later for analysis of $\mathrm{Cd}$ content. After sorting, containers were frozen and shipped to the University of Guelph, where they were stored at $-20^{\circ} \mathrm{C}$ until analysis. Representative specimens of invertebrates were preserved in $4 \%$ formalin and identified by Dr C. T. Shih, National Museum of Canada, Ottawa.

Arctic cod Boreogadus saida were collected by seine in October 1983 at Arctic Bay, northern Baffin Island. Fish were individually sealed in Whirl-pak bags and frozen within $2 \mathrm{~h}$ of capture. Identification was confirmed by Mr C. Renaud, Ichthyology Section, National Museum of Canada.

Sample preparation. Glassware was cleaned by soaking in a dilute solution of a commercial preparation of phosphoric acid (Servac, Wyandotte Corp.), then soaking in double deionized water for $24 \mathrm{~h}$, in $5 \%$ $\mathrm{HNO}_{3}$ for $24 \mathrm{~h}$, and double deionized water for $24 \mathrm{~h}$. Before weighing, glassware was heated overnight at $80^{\circ} \mathrm{C}$ and cooled in a dessicator. Plastic Petri dishes and vials for sample storage were soaked for $24 \mathrm{~h}$ in $5 \%$ $\mathrm{HNO}_{3}$ and $24 \mathrm{~h}$ in double deionized water. Since $\mathrm{Cd}$ is used as a stabilizer in some plastics (Dixon 1981), only clear, linear polyethylene was used to store samples. Organisms were handled only with stainless steel or teflon-coated instruments.

Before analysing for Cd content, invertebrates were thawed, identified to species, and usually measured for total body length (for crustaceans, from the posterior tip of the telson to the anterior tip of the eye). Both wet and dry weights were measured in 1983 and 1984 and those samples are the main results reported here. Only wet weight was measured for most of the 1982 samples, however samples of some organisms such as Mysis litoralis were pooled in the field so that freezing altered the water content, and Cd concentrations were based on dry weight. Because of this inconsistency, samples from 1982 are used primarily to support the data from the other 2 yr. For very small organisms, individuals of equal length were grouped together to allow accuracy in weighing as well as sufficient $\mathrm{Cd}$ to exceed detection limits. Approximately 10 small Parathemisto libellula ( 3 to $4 \mathrm{~mm}$ total length) were grouped together, while up to 50 small Gammarus setosus (taken at Kaujuitoq in 1984 from the brood pouch of females) made up a single sample. Mysids, chaetognaths, medusae and ctenophores could not be 
separated as individuals because of the damage during freezing and were grouped to species in the field, and subsamples taken for analysis. Individual arctic cod were weighed and measured for total length, dried for $48 \mathrm{~h}$ at $80^{\circ} \mathrm{C}$ and re-weighed. Cod livers collected from harp seal stomachs were dissected from the partially digested fish, dried for $48 \mathrm{~h}$ at $70^{\circ} \mathrm{C}$ and digested as outlined below.

Samples were digested using the wet ashing technique of Wagemann et al. (1983) with slight modification. Concentrated reagent grade $\mathrm{HNO}_{3}$ was added to the sample and allowed to sit overnight or until foaming stopped, heated gently until almost dry and further $\mathrm{HNO}_{3}$ added. The heating and $\mathrm{HNO}_{3}$ additions were repeated until little residue remained. A final addition of $\mathrm{HNO}_{3}$ included enough concentrated $\mathrm{H}_{2} \mathrm{SO}_{4}$ to bring the final dilution of the digest to 2 to $8 \% \mathrm{H}_{2} \mathrm{SO}_{4}$. The sample was heated until charring occurred, cooled, and clarified using 30 or $50 \% \mathrm{H}_{2} \mathrm{O}_{2}$. Charring and clarification were repeated until charring no longer occurred. Two blank digests were included with the samples and given the same quantity of acids and peroxide as the sample. After final charring, the sample was clarified and brought to volume (e.g. $10.0 \mathrm{ml}$ or $25.0 \mathrm{ml}$ ) with double deionized water. Samples were stored in glass vials for $1 \mathrm{wk}$ or less, or in vials of linear polyethylene for longer times.

All 1983 and 1984 samples were analysed using a Scintrex AAZ-2 spectrophotometer with electrothermal atomization and Zeeman modulation background correction. The detection limit, defined as 3 times the standard deviation of the blanks, was ca $0.50 \mathrm{ng} \mathrm{ml}^{-1}$, giving a working range of 1.00 to $5.00 \mathrm{ng} \mathrm{Cd} \mathrm{ml}^{-1}$ using $10 \mu \mathrm{l}$ samples. The atomization program was: drying time 10 to $15 \mathrm{~s}$; ashing $250^{\circ} \mathrm{C}$ for $30 \mathrm{~s}$; atomization 1300 to $1350^{\circ} \mathrm{C}$ for $3 \mathrm{~s}$. The atomization cycle and subsequent calculation of standard curves and concentrations were conducted by an Apple II microcomputer using software purchased from Scintrex Corp., Toronto, Canada. Samples collected in 1982 were analysed with a Varian AA6 in flameless mode using CRA-90 carbon rod attachment and $\mathrm{H}_{2}$-deuterium background correction. The atomization cycle was: drying $100^{\circ} \mathrm{C}$ for $20 \mathrm{~s}$; ashing $350^{\circ} \mathrm{C}$ for $30 \mathrm{si}$ atomization $1200^{\circ} \mathrm{C}$ for $3 \mathrm{~s}$ at a ramp rate of 225 . Working range was 5.0 to $50.0 \mathrm{ng} \mathrm{Cd}$ $\mathrm{ml}^{-1}$ for a sample size of $2 \mu \mathrm{l}$. All standards were made by dilution of a $1.00 \mathrm{mg} \mathrm{Cd} \mathrm{g}^{-1}$ standard (Fisher Scientific) in a solution of the appropriate $\mathrm{H}_{2} \mathrm{SO}_{4}$ concentration $(2$ or $8 \%$ ).

Total $\mathrm{Cd}$ in the digest was calculated as concentration in $\mathrm{ng} \mathrm{ml} \mathrm{m}^{-1} \times$ number of $\mathrm{ml}$ in the digest. The mean total Cd (ng) in the blanks was then subtracted, giving the amount of $\mathrm{Cd}$ present in the organisms. This value was divided by the number of individuals in the sample to produce the body burden. Cd concentration was calculated as body burden (ng $\mathrm{Cd}$ ) divided by body weight ( $m g$; wet and dry weight when possible). Concentration in grouped individuals was derived by dividing the total $\mathrm{Cd}$ in the digest by the sample weight, to avoid errors caused by rounding off. Each digest was considered to be one observation, for both large and small organisms, in subsequent statistical analyses.

Analytical accuracy and precision. Blank samples, spiked blank samples, and NBS bovine liver standard 1577 a (National Bureau of Standards, Washington, D.C.) were analysed at the same time as field samples. Blank and spiked blank vials showed no loss of $\mathrm{Cd}$ by volatilization with the heating during digestion (Table 1). Contamination from the reagent grade chemicals remained constant at 3 to $5 \mathrm{ng} \mathrm{Cd}$ per digest. The contamination measured in the blank digests was assumed to be constant through each sample set (generally 43 samples per set) and was subtracted from the total $\mathrm{Cd}$ measured in each digest. For the $3 \mathrm{yr}$ of samples, only 3 sets of samples were discarded because of apparent contamination.

Measured $C d$ in NBS standard bovine liver was 0.41 (SD 0.04) $\mu \mathrm{g} \mathrm{Cd} \mathrm{g}{ }^{-1}$ dry weight $(n=5$ ) or ca $94 \%$ of the published value of $0.44 \pm 0.06 \mu \mathrm{g} \mathrm{Cd} \mathrm{g}{ }^{-1} \mathrm{dry}$ weight. The standard deviation was $9.6 \%$ of the mean. Precision, defined here as the standard error of the mean, averaged $4.3 \%$. Interference during the atomic absorption procedure was tested by adding spikes of 0 to $400 \mathrm{ng} \mathrm{Cd}$ to a homogenized sample of Parathemisto libellula. The resulting regression of meas-

Table 1. Summary of tests of analytical accuracy and precision using the Scintrex AAZ-2 atomic absorption spectrophotometer to measure $\mathrm{Cd}$. All concentrations are in $\mathrm{ng} \mathrm{g}^{-1}$, followed by standard deviations. Expected concentration for NBS bovine liver is based on a dry weight concentration of $440 \mathrm{ng}^{-1}$

\begin{tabular}{|c|c|c|c|}
\hline Sample & $\begin{array}{c}\text { Expected concentration } \\
\text { in digest }\end{array}$ & $\begin{array}{c}\text { Observed concentration } \\
\text { in digest }\end{array}$ & $\begin{array}{l}\text { Number of } \\
\text { measurements }\end{array}$ \\
\hline Blank & 0.00 & $0.40 \pm 0.25$ & 4 \\
\hline Blank with spike of $20.0 \mathrm{ng} \mathrm{Cd}$ & 2.00 & $2.09 \pm 0.23$ & 5 \\
\hline Blank with spike of $100.0 \mathrm{ng} \mathrm{Cd}$ & 10.0 & $10.1 \pm 0.30$ & 3 \\
\hline NBS bovine liver standard 1577 a & $2.26 \pm 0.42$ & $2.21 \pm 0.57$ & 5 \\
\hline
\end{tabular}


ured versus nominal (or spiked) Cd showed a direct relationship with a slope of $1.07\left(r^{2}=0.98\right)$, indicating full recovery of the spikes.

If similar samples (i.e. the same species from the same area but different years) yielded different cadmium concentrations on the 2 spectrophotometers, values determined on the Scintrex machine were considered more reliable because of Zeeman background correction and the opportunity to inspect printed absorption curves. For example, 1982 measurements on the amphipod Anonyx sarsi were discarded because of high variability, possibly caused by interference during the absorption phase. Subsequent measurements of $A$. sarsi with the Scintrex machine were relatively uniform. Consistency of $\mathrm{Cd}$ concentrations was also tested by comparing wet weight concentrations of 3 species collected from the same area at Cape Hatt in 1982 and 1983. There was no significant difference observed for Parathemisto libellula $\left(4.07 \mu \mathrm{g} \mathrm{g}^{-1} \pm \mathrm{SD}\right.$ 1.38 to $12.04, n=37 ; 5.70 \mu \mathrm{g} \mathrm{g}^{-1} \pm \mathrm{SD} 2.58$ to 12.56 , $n=59)$, or for Gammarus setosus $\left(0.08 \mu \mathrm{g} \mathrm{g}^{-1} \pm \mathrm{SD}\right.$ 0.04 to $0.19, n=19 ; 0.07 \mu g^{-1} \pm \mathrm{SD} 0.04$ to $0.15, n=$ 41). For Boeckismus edwardsi the mean wet-weight concentrations for the $2 \mathrm{yr}$ proved to be significantly different $\left(0.15 \mu \mathrm{g} \mathrm{g}^{-1} \pm \mathrm{SD} 0.08\right.$ to $0.29, n=36 ; 0.23 \mu \mathrm{g}$ $\mathrm{g}^{-1} \pm \mathrm{SD} 0.17$ to $\left.0.31, n=24\right)$.

Statistical analysis of results used commercially available statistical packages. Most regressions and residual analyses were done using BMDP (Dixon et al. 1983), while all ANOVA tests and comparisons of means were conducted using SAS (SAS Institute, Cary,
North Carolina, USA 1982). A statistically significant difference was considered to exist if $p \leq 0.05$.

\section{RESULTS}

The major groups of organisms collected, their diets, and range of body lengths are given in Table 2. Four of the species in which individual organisms could be analysed (Parathemisto libellula, Anonyx sarsi, Boreogadus saida, Gammarus setosus) showed continuous ranges of body size from young of the year ( $\mathrm{Y}-\mathrm{O}-\mathrm{Y}$ ) to adults, while samples of Mysis litoralis, Boeckismus edwardsi and the Kaujuitoq sample of $G$. setosus showed distinct bimodal distributions of size. The larger $M$. litoralis (mean length of $24.8 \mathrm{~mm}$ ) contained reproductively mature males with extended pleopods and females with fully developed brood chambers. The size classes of $G$. setosus from Kaujuitoq consisted of gravid females (mean length of $26.7 \mathrm{~mm}$ ) and a separate $\mathrm{Y}-\mathrm{O}-\mathrm{Y}$ class of 3 to $4 \mathrm{~mm}$, collected from brood pouches of females.

For those samples involving pooled individuals, average $\mathrm{Cd}$ concentrations ranged from $0.17 \mathrm{\mu g} \mathrm{g}^{-1}$ for Mysis litoralis to $6.31 \mathrm{\mu g} \mathrm{g}^{-1}$ in a homogenized sample of the hyperiid amphipod Parathemisto libellula taken from the gut of harp seals (Table 3, dry weights). The 3 samples of epontic community were not significantly different from each other in cadmium concentration, nor were the samples of copepods, ctenophores or chaetognaths. The averages in Table 3 were calculated

Table 2. Summary of major groups and sizes of organisms collected, with their habitats, diets and sites of collection in the Northwest Territories

\begin{tabular}{|c|c|c|c|c|}
\hline Species/Group & Site & Habitat & Diet & Body length range $(\mathrm{mm})$ \\
\hline Epontic community & Kaujuitoq & Underice surface & - & - \\
\hline Copepods & $\begin{array}{l}\text { Cape Hatt } \\
\text { Kaujuitoq }\end{array}$ & $\begin{array}{l}\text { Pelagic } \\
\text { Pelagic }\end{array}$ & $\begin{array}{l}\text { Herbivore } \\
\text { Herbivore }\end{array}$ & $\begin{array}{l}0.1-6.0 \\
0.1-7.0\end{array}$ \\
\hline Mysis litoralis & Cape Hatt & Benthic-pelagic & Detritivore & $12.0-28.0^{\circ}$ \\
\hline \multicolumn{5}{|l|}{ Amphipods } \\
\hline Gammarus setosus & $\begin{array}{l}\text { Cape Hatt } \\
\text { Kaujuitoq }\end{array}$ & $\begin{array}{l}\text { Intertidal zone } \\
\text { Underice surface }\end{array}$ & $\begin{array}{l}\text { Herbivore } \\
\text { Herbivore }\end{array}$ & $\begin{array}{l}9.5-35.0 \\
3.5-36.0^{\circ}\end{array}$ \\
\hline Onisimus glacialis & Cape Hatt & Pelagic & Omnivore & $4.0-15.0$ \\
\hline Anonyx sarsi & Cape Hatt & Benthic & Omnivore (scavenger) & $7.9-41.0$ \\
\hline Parathemisto libellula & Cape Hatt & Pelagic & Carnivore & $\begin{array}{l}4.5-37.0 \\
7 .-25.1\end{array}$ \\
\hline P. abyssorum & $\begin{array}{l}\text { Kaujuitoq } \\
\text { Kaujuitoq }\end{array}$ & $\begin{array}{l}\text { Pelagic } \\
\text { Pelagic }\end{array}$ & $\begin{array}{l}\text { Carnivore } \\
\text { Carnivore }\end{array}$ & $\begin{array}{r}2.7-25.1 \\
13.0-16.0\end{array}$ \\
\hline Hyperoche medusarum & Kaujuitoq & Pelagic & Carnivore & $4.0-9.0$ \\
\hline Boeckismus edwardsi & Cape Hatt & Benthic & Omnivore (scavenger) & $5.0-14.0^{\circ}$ \\
\hline Fish & & & & \\
\hline Boreogadus saida & Arctic Bay & Pelagic & Carnivore & $70.0-168.0$ \\
\hline
\end{tabular}


Table 3. Mean Cd concentration of pooled organisms collected at Cape Hatt, Kaujuitoq and Grise Fiord. Sample size (n) refers to number of subsamples

\begin{tabular}{|c|c|c|c|c|}
\hline Sample & Site & Year & $\begin{array}{c}\text { Cd concentration } \\
\left(\mu \mathrm{g} \mathrm{g}^{-1} \text { dry weight }\right) \\
( \pm \mathrm{SD})\end{array}$ & $n$ \\
\hline Phytoplankton & Cape Hatt & 1983 & $4.74(3.77-5.96)$ & 5 \\
\hline $\begin{array}{l}\text { Epontic community } \\
\text { Filtered } \\
<64 \mu \mathrm{m} \text { fraction } \\
>64 \mu \mathrm{m} \text { fraction }\end{array}$ & $\begin{array}{l}\text { Kaujuitoq } \\
\text { Kaujuitoq } \\
\text { Kaujuitoq }\end{array}$ & $\begin{array}{l}1983 \\
1984 \\
1984\end{array}$ & $\begin{array}{l}1.84(0.75-4.56) \\
1.93(1.12-3.32) \\
1.47(1.09-1.97)\end{array}$ & $\begin{array}{r}7 \\
18 \\
8\end{array}$ \\
\hline Copepods & $\begin{array}{l}\text { Kaujuitoq } \\
\text { Cape Hatt } \\
\text { Kaujuitoq }\end{array}$ & $\begin{array}{l}1983 \\
1983 \\
1984\end{array}$ & $\begin{array}{l}1.67(1.28-2.18) \\
2.16(1.81-2.58) \\
2.37(2.00-2.82)\end{array}$ & $\begin{array}{r}12 \\
5 \\
4\end{array}$ \\
\hline $\begin{array}{l}\text { Pteropods } \\
\text { Clione limacina }\end{array}$ & Cape Hatt & 1983 & $1.86(1.67-2.07)$ & 3 \\
\hline Ctenophores & $\begin{array}{l}\text { Cape Hatt } \\
\text { Kaujuitoq } \\
\text { Kaujuitoq }\end{array}$ & $\begin{array}{l}1983 \\
1983 \\
1984\end{array}$ & $\begin{array}{l}0.95(0.79-1.14) \\
0.62(0.40-0.96) \\
1.01(0.95-1.05)\end{array}$ & $\begin{array}{l}7 \\
5 \\
2\end{array}$ \\
\hline $\begin{array}{l}\text { Mysids } \\
\text { Mysis litoralis }\end{array}$ & Cape Hatt & 1982 & $0.17(0.14-0.20)$ & 9 \\
\hline $\begin{array}{l}\text { Amphipods } \\
\text { Parathemisto libellula }\end{array}$ & Grise Fiord & 1983 & $6.31(5.31-7.50)$ & 10 \\
\hline Chaetognaths & $\begin{array}{l}\text { Cape Hatt } \\
\text { Kaujuitoq }\end{array}$ & $\begin{array}{l}1983 \\
1984\end{array}$ & $\begin{array}{l}1.00(0.66-1.49) \\
1.31(0.95-1.80)\end{array}$ & $\begin{array}{r}11 \\
4\end{array}$ \\
\hline $\begin{array}{l}\text { Fish } \\
\text { Boreogadus saida (livers) }\end{array}$ & Grise Fiord & 1983 & $1.14(0.77-1.68)$ & 10 \\
\hline
\end{tabular}

as arithmetic means of untransformed data, and sample size refers to the number of subsamples analysed.

Mean dry-weight concentrations for measurements on individual organisms were calculated as geometric means to normalize the residuals (Table 4). The means ranged from consistently low values in Gammarus setosus from Z-lagoon at Cape Hatt and in the mysid Mysis litoralis (0.45 and $0.29 \mu \mathrm{g} \mathrm{g}^{-1}$, respectively), to high values of 14 to $16 \mu \mathrm{g} \mathrm{g}^{-1}$ in some samples of the hyperiid amphipods Parathemisto spp. and Hyperoche medusarum. Cd concentrations did not differ significantly among samples of a given species, except that $G$. setosus showed a difference for 2 adjacent bays at Cape Hatt in 1983 (Z-lagoon, $0.45 \mu \mathrm{g} \mathrm{g}^{-1}$; Bay 12, $9.57 \mu \mathrm{g} \mathrm{g}^{-1}$ ) and also between each of the Cape Hatt samples and the one collected at Kaujuitoq $(3.07 \mu \mathrm{g}$ $\left.\mathrm{g}^{-1}\right)$.

For all the dry-weight $\mathrm{Cd}$ concentrations of individuals or pooled samples of the same organisms (Tables 3 \& 4), Scheffe's multiple-range test showed no significant difference between sampling sites (Cape Hatt versus Kaujuitoq), seasons (summer versus spring) and year of sampling (1982 versus 1983 at Cape Hatt, 1983 versus 1984 at Kaujuitoq), with the single exception of Gammarus setosus which was accordingly treated as
3 discrete samples. A grand mean for each species or group was calculated by pooling all data from all samples. Those grand means are compared with summarized data from other regions in Table 5. Means from the present study are categorized into trophic levels in Fig. 2, based on the diets (i.e. level $1=$ primary producers, $2=$ herbivores, $2.5=$ omnivores and scavengers, $3=$ carnivores, $4=$ secondary carnivores). Despite the similarity in the diets of hyperiid amphipods and arctic cod (see below), the latter have been placed in a marginally higher trophic level because they consume hyperiid amphipods.

There were differences between the grand mean $\mathrm{Cd}$ concentrations of most species (Table 6), as determined by Scheffe's multiple-range test on all pooled species data or grand means, after first removing Parathemisto abyssorum and the pteropod Clione limacina because of small sample sizes $(n=3)$. The hyperiid amphipods P. abyssorum, P. libellula and Hyperoche medusarum had the highest $\mathrm{Cd}$ concentrations, followed by the Bay 12 sample of the amphipods Gammarus setosus and Onisimus glacialis, a pelagic omnivore. The high concentrations in $P$. libellula were consistent through all samples collected, despite the higher proportion of Y-O-Y sampled at Kaujuitoq in both 1983 and 1984 (i.e. 


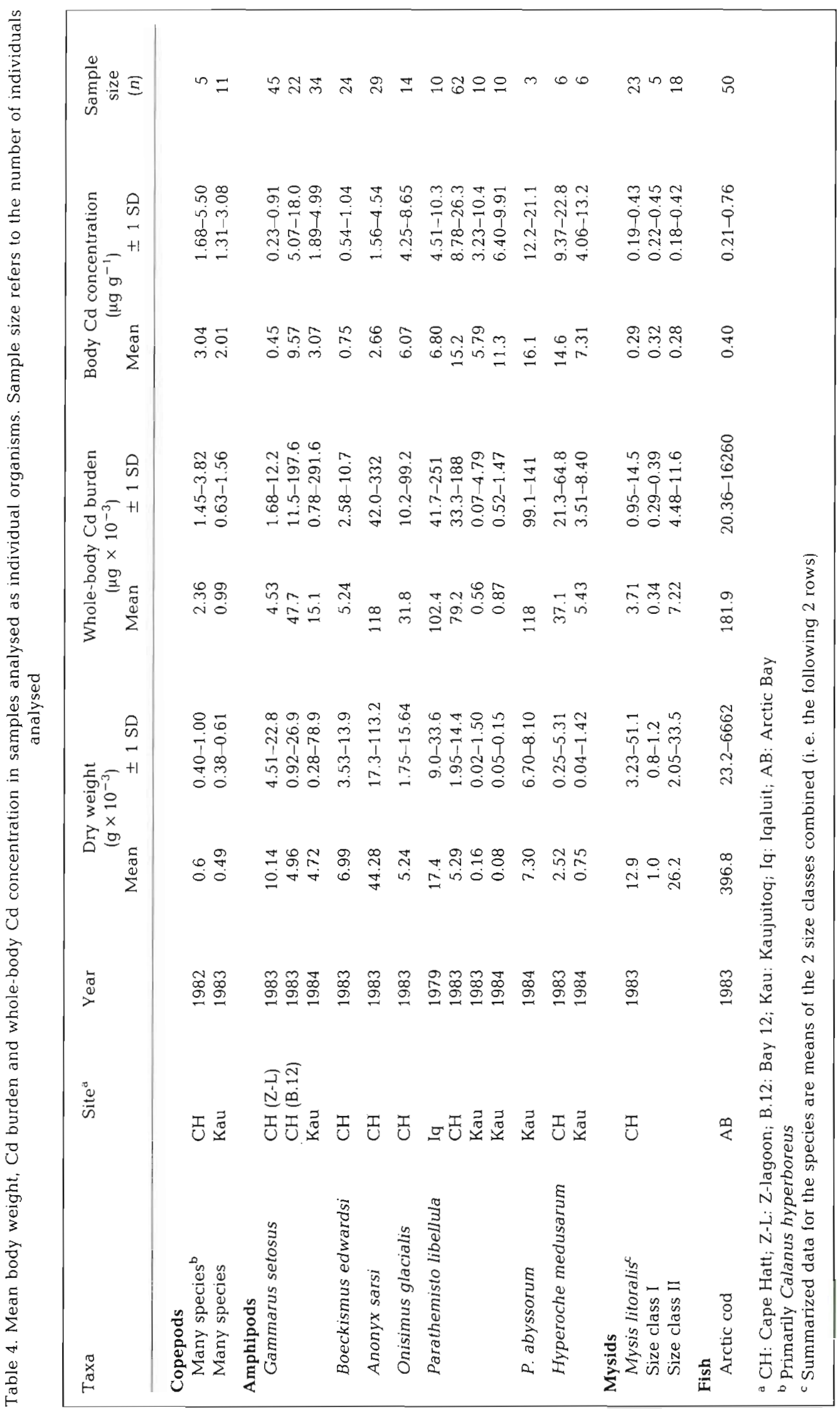


Table 5. Comparison of Cd data from present study with $\mathrm{Cd}$ values from the literature. N.W.T.: Northwest Territories

\begin{tabular}{|c|c|c|c|c|c|}
\hline Source & Area & $n$ & $\begin{array}{c}\text { Mean } \\
\text { concentration } \\
\left(\mu g g^{-1} \text { dry weight }\right) \\
( \pm 1 \text { SD })\end{array}$ & Range & Reference \\
\hline \multicolumn{6}{|l|}{ Water (surface $; \mu g ~^{-1}$ ) } \\
\hline Dissolved & N. Atlantic & & 0.063 & & Eaton (1976) \\
\hline Total & North Sea & & 0.024 & & Danielssen et al. (1985) \\
\hline Dissolved & North Sea & & 0.02 & $0.008-0.025$ & Kremling (1985) \\
\hline Total & Central Arctic & & 0.034 & & Moore (1981) \\
\hline Dissolved & Strathcona Sound, N.W.T. & & 0.040 & & Thomas et al. (1983) \\
\hline Dissolved & E. Davis Strait, N.W.T & & 0.030 & & Campbell \& Yeats (1982) \\
\hline Dissolved & Smith Sound, N.W.T & & 0.040 & & Campbell \& Yeats (1982) \\
\hline Dissolved & Lancaster Sound, N.W.T & & 0.060 & & Campbell \& Yeats (1982) \\
\hline Dissolved & Baffin Bay, N.W.T & & - & $0.05-0.06$ & Campbell \& Yeats (1982) \\
\hline Total & Baffin Bay, N.W.T & & - & $0.02-0.06$ & Campbell \& Loring (1980) \\
\hline \multicolumn{6}{|l|}{ Phytoplankton } \\
\hline Grouped sample (>64 $\mu \mathrm{m})$ & California & 22 & $11.72 \pm 6.00$ & $2.2-24.7$ & Martin et al. (1976) \\
\hline Grouped sampie $(>70 \mu \mathrm{m})$ & Califomia & - & - & $0.5-7.0$ & Knauer \& Martin (1973) \\
\hline Grouped sample (>64 $\mu \mathrm{m})$ & Baja California & 11 & $14.0 \pm 4.77$ & $2.8-17.9$ & Bruland et al. (1978) \\
\hline Grouped sample (>64 $\mu \mathrm{m})$ & Northeast Pacific & - & - & $2-5$ & Martin \& Broenkow (1975) \\
\hline Grouped sample $(>64 \mu \mathrm{m})$ & Baja California & 22 & 13.6 & $4.2-20.9$ & Martin \& Broenkow (1975) \\
\hline Grouped sample (>64 $\mu \mathrm{m}$ ) & Pond Inlet, N.W.T & 5 & $4.74(3.77-5.96)$ & - & Present study \\
\hline $\begin{array}{l}\text { Epontic community } \\
(>0.45 \mu \mathrm{m})\end{array}$ & Kaujuitoq, N.W.T & 7 & $1.84(0.75-4.56)$ & - & Present study \\
\hline $\begin{array}{l}\text { Epontic community } \\
\qquad(>0.45 \mu \mathrm{m}<64 \mu \mathrm{m})\end{array}$ & Kaujuitoq, N.W.T. & 18 & $1.93(1.12-3.32)$ & - & Present study \\
\hline $\begin{array}{l}\text { Epontic community } \\
\qquad(>64 \mu \mathrm{m})\end{array}$ & Kaujuitoq, N.W.T. & 8 & $1.47(1.09-1.97)$ & - & Present study \\
\hline \multicolumn{6}{|l|}{ Copepods } \\
\hline Grouped sample & California & 10 & $4.3 \pm 1.75$ & $1.6-7.5$ & Martin \& Knauer (1973) \\
\hline Grouped sample & Pacific Ocean & 28 & $2.30 \pm 1.57$ & $0.6-6.5$ & Martin \& Knauer (1973) \\
\hline Grouped sample & Baja California & - & 15.2 & - & Martin \& Broenkow (1975) \\
\hline Grouped sample & Baffin Island & 2 & $2.8 ; 5.0$ & - & Bohn \& McElroy (1976) \\
\hline Grouped sample & Loire Estuary, France & 2 & $0.77 ; 0.83$ & $0.39-1.15$ & Amiard-Triquet et al. (1980) \\
\hline Grouped sample & North Pacific & 13 & $7.68 \pm 6.54$ & $0.97-18.13$ & Hamanaka \& Mishima (1981) \\
\hline Grouped sample & Bering Sea & 6 & $6.62 \pm 2.90$ & - & Hamanaka \& Mishima (1981) \\
\hline $\begin{array}{l}\text { Zooplankton (mostly } \\
\text { copepods) }\end{array}$ & Baltic Sea & 19 & - & $0.20-4.6$ & Szefer et al. (1985) \\
\hline Grouped sample & E. Cdn. Arctic & 21 & $1.94(1.40-2.69)$ & $0.69-3.80$ & Present study \\
\hline Labidocera acutifrons & Atlantic Ocean & 1 & 9.8 & - & Leatherland et al. (1973) \\
\hline Anomalocera patersoni & Mediterranean Sea & - & $1.51 \pm 0.72$ & - & Polikarpov et al. (1979) \\
\hline Calanus plumchrus & Bering Sea & 5 & $1.66 ; 12.69$ & - & Hamanaka \& Tsujita (1981) \\
\hline C. plumchrus & N. Pacific Ocean & 8 & 8.82 & $3.09-14.55$ & Hamanaka \& Tsujita (1981) \\
\hline C. plumchrus & Okhotsk Sea & 3 & 4.94 & $4.67 ; 5.21$ & Hamanaka \& Tsujita (1981) \\
\hline C. cristatus & N. Pacific Ocean & 2 & 2.35 & - & Hamanaka \& Tsujita (1981) \\
\hline C. glacialis & Okhotsk Sea & 11 & $6.63 \pm 4.19$ & $1.66-14.55$ & Hamanaka \& Tsujita (1981) \\
\hline \multicolumn{6}{|l|}{ Chaelognaths } \\
\hline Grouped sample & N. Baffin Island & 2 & $1.2 ; 1.3$ & - & Bohn \& McElroy (1976) \\
\hline Grouped sample & N. Pacific Ocean & 2 & $1.49 \pm 0.03$ & - & Hamanaka \& Mishima (1981) \\
\hline Grouped sample & E. Cdn. Arctic & 15 & $1.07(0.72-1.59)$ & $0.54-1.78$ & Present study \\
\hline \multicolumn{6}{|l|}{ Mysids } \\
\hline Eucopia sculpticauda & N. Atlantic & 2 & 2.0 & - & Leatherland et al. (1973) \\
\hline Grouped sample & Loire Estuary, France & 2 & $0.06 ; 0.09$ & - & Amiard-Triquet et al. (1980) \\
\hline Grouped sample & California & - & 0.432 & - & Schafer et al. (1982) \\
\hline Mysis litoralis & Cape Hatt, N.W.T & 32 & $0.25(0.16-0.38)$ & $0.13-0.60$ & Present study \\
\hline \multicolumn{6}{|l|}{ Amphipods } \\
\hline Lysianassidae & Baffin Island & 1 & 7.0 & - & Bohn \& McElroy $(1976)$ \\
\hline Gammarus zaddachi & Loire Estuary, France & 2 & $0.22 ; 0.28$ & - & Amiard-Triquet et al. (1980) \\
\hline G. setosus & Z-lag. Cape Hatt & 45 & $0.45(0.23-0.91)$ & $0.08-2.04$ & Present study \\
\hline G. setosus & Bay 12, Cape Hatt & 35 & $9.57(5.07-18.0)$ & $2.68-26.7$ & Present study \\
\hline
\end{tabular}


Table 5 (continued)

\begin{tabular}{|c|c|c|c|c|c|c|}
\hline Source & Area & $n$ & \multicolumn{2}{|c|}{$\begin{array}{c}\text { Mean } \\
\text { concentration } \\
\left.\text { (ug g } g^{-1} \text { dry weight }\right) \\
( \pm 1 \mathrm{SD})\end{array}$} & Range & Reference \\
\hline \multicolumn{7}{|l|}{ Amphipods } \\
\hline G. setosus & Kaujuitoq, N.W.T & 22 & \multicolumn{2}{|c|}{$3.07(1.89-4.99)$} & $1.05-9.15$ & Present study \\
\hline Boeckismus edwardsi & Cape Hatt, N.W.T & 24 & \multicolumn{2}{|c|}{$0.75(0.54-1.04)$} & $0.40-1.38$ & Present study \\
\hline Anonyx sarsi & Cape Hatt, N.W.T & 29 & \multicolumn{2}{|c|}{$2.66(1.56-4.54)$} & $0.82-6.99$ & Present study \\
\hline Onisimus glacialis & Cape Hatt, N.W.T & 14 & \multicolumn{2}{|c|}{$6.07(4.25-8.65)$} & $2.69-11.8$ & Present study \\
\hline $\begin{array}{l}\text { Mixed sample (mostly } \\
\text { Parathemisto sp.) }\end{array}$ & N. Pacific Ocean & 13 & \multicolumn{2}{|c|}{$21.44 \pm 10.78$} & $1.74-36.93$ & Hamanaka \& Mishima (1981) \\
\hline Parathemisto libellula & Bering Sea & 11 & \multicolumn{2}{|c|}{5.27} & $(4.72 ; 6.24)$ & Hamanaka \& Mishima (1981) \\
\hline P. libellula & Bering Sea & 3 & \multicolumn{2}{|c|}{6.68} & $5.37-7.98$ & Hamanaka \& Tsujita (1981) \\
\hline P. libellula & Bering Sea & 18 & \multicolumn{2}{|c|}{$9.07 \pm 7.71$} & $3.35-33.3$ & Hamanaka \& Ogi (1984) \\
\hline P. libellula & E. Cdn. Arctic & 97 & \multicolumn{2}{|c|}{$11.4(5.96-21.8)$} & $2.80-42.5$ & Present study \\
\hline P. pacifica & N. Pacific Ocean & 6 & \multicolumn{2}{|c|}{$10.30 \pm 5.64$} & $4.16-14.50$ & Hamanaka \& Tsujita (1981) \\
\hline P. pacifica & Bering Sea & 1 & \multicolumn{2}{|c|}{6.72} & - & Hamanaka \& Tsujita (1981) \\
\hline P. japonica & Okhotsk Sea & 1 & \multicolumn{2}{|c|}{2.83} & - & Hamanaka \& Tsujita (1981) \\
\hline P. abyssorum & Kaujuitoq, N.T.W. & 3 & \multicolumn{2}{|c|}{$16.1(12.3-21.1)$} & $11.8-19.5$ & Present study \\
\hline Hyperia galba & Bering Sea & 2 & \multicolumn{2}{|c|}{$11.34 \pm 0.41$} & - & Hamanaka \& Tsujita (1981) \\
\hline Hyperoche medusarum & E. Cdn. Arctic & 12 & \multicolumn{2}{|c|}{$10.3(5.59-19.1)$} & $3.48-29.5$ & Present study \\
\hline \multicolumn{7}{|l|}{ Fish } \\
\hline Gadus morhua & Norway & 2 & Muscle & 0.01 & - & Lande (1977) \\
\hline G. morhua & Malvik, Norway & 4 & Muscle & 0.02 & - & Lande (1977) \\
\hline G. morhua & Gulf of Bothnia & - & Liver & - & $0.06-0.18$ & Pertilla et al. (1982) \\
\hline G. morhua & Gulf of Bothnia & 9 & Muscle & - & $0.008-0.072$ & Miettinen \& Verta (1978) \\
\hline G. morhua & Baltic Sea & - & Muscle & - & $0.008-0.20^{a}$ & Brugmann (1981) \\
\hline \multirow[t]{2}{*}{ Boreogadus saida } & \multirow{2}{*}{$\begin{array}{l}\text { Strathcona Sound, } \\
\text { N.W.T }\end{array}$} & 15 & \multirow{2}{*}{$\begin{array}{l}\text { Liver } \\
\text { Whole fish }\end{array}$} & $0.72 \pm 0.37$ & $0.35-1.6$ & \multirow[t]{2}{*}{ Bohn \& McElroy (1976) } \\
\hline & & 47 & & $0.62 \pm 0.22$ & $0.26-1.5$ & \\
\hline B. saida & $\begin{array}{l}\text { Strathcona Sound, } \\
\text { N.W.T }\end{array}$ & 50 & Whole body & $\begin{array}{c}0.40 \\
(0.21-0.76)\end{array}$ & $0.13-1.51$ & Present study \\
\hline B. saida & Grise Fiord, N.W.T. & 6 & Liver & $4(0.77-1.68)$ & $0.69-1.86$ & Present study \\
\hline Clupea harengus & Malvik, Norway & 2 & Muscle & 0.01 & - & Lande (1977) \\
\hline C. harengus & Gulf of Bothnia & 20 & Muscle & $0.024 \pm 0.012$ & - & Pertilla et al. (1982) \\
\hline C. harengus & Baltic Sea & - & Muscle & - & $0.008-0.800$ & Brugmann (1981) \\
\hline Baltic herring & Gulf of Bothnia & 147 & Muscle & - & $0.05-0.17^{\mathrm{a}}$ & Miettinen \& Verta (1978) \\
\hline Sprat & Gulf of Bothnia & 32 & Muscle & - & $0.07-0.08^{a}$ & Miettinen \& Verta (1978) \\
\hline Perch & Gulf of Bothnia & 198 & Muscle & - & $0.01-0.14^{\mathrm{a}}$ & Miettinen \& Verta (1978) \\
\hline Myoxocephalus scorpius & Strathcona Sound, & 3 & Muscle & $1.4 \pm 0.3$ & $1.0-1.6$ & Bohn \& Fallis (1978) \\
\hline & N.W.T. & 61 & Liver & $4.1 \pm 3.1$ & $0.9-16.0$ & \\
\hline Salvelinus alpinus & $\begin{array}{l}\text { Strathcona Sound, } \\
\text { N.W.T }\end{array}$ & 3 & Liver & $2.0 \pm 0.3$ & $1.6-2.3$ & Bohn \& Fallis (1978) \\
\hline
\end{tabular}

mean body weights of 0.16 and $0.08 \mathrm{mg}$, respectively). The 1983 sample consisted of 6 digests, each with 10 small (3 to $4 \mathrm{~mm}$ ) $P$. libellula, and a single digest with one adult, yet it still showed a mean concentration of $5.79 \mu \mathrm{g} \mathrm{g}^{-1}$.

Arctic cod contained the second lowest whole-body concentration $\left(0.40 \mu \mathrm{g} \mathrm{g}^{-1}\right)$ among the 17 groups tested and was significantly lower than both the copepods and hyperiid amphipods which are a major part of its diet. Arctic cod was not significantly different from Mysis litoralis, another of its food items.
The variability of $\mathrm{Cd}$ content between the groups of organisms can also be illustrated by comparing wholebody burden of $\mathrm{Cd}$ (Table 4). Arctic cod had the highest values, with a mean burden of $182 \mathrm{ng} \mathrm{Cd}(n=50)$ and a maximum of $3430 \mathrm{ng} \mathrm{Cd}$ in a single fish. However, since that fish weighed $8.2 \mathrm{~g}$ (dry), its concentration was only $0.42 \mu \mathrm{g} \mathrm{g}^{-1}$. The next highest burdens were found in the amphipods Anonyx sarsi, Parathemisto abyssorum and $P$. libellula which had mean values of 118,118 and $79.2 \mathrm{ng} \mathrm{Cd}$ respectively. One specimen of $P$. libellula collected at Cape Hatt in 1983 contained 


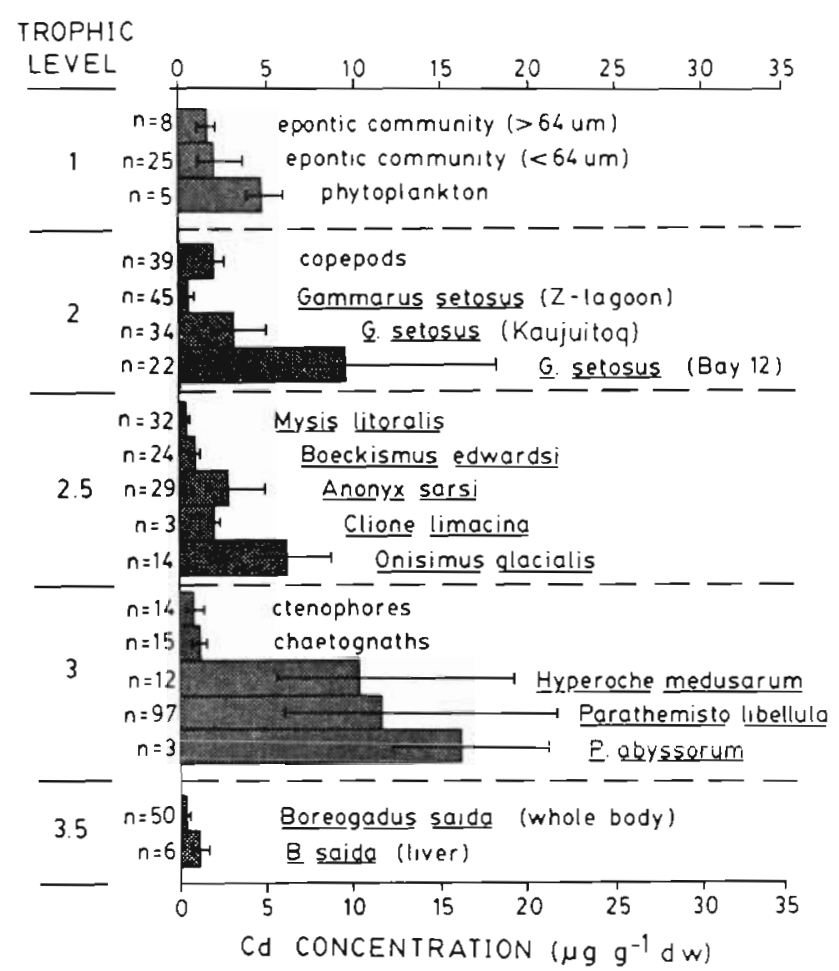

Fig. 2. Trophic level of organisms versus mean $\mathrm{Cd}$ concentration of the species reported in Table 5. Values for Gammarus setosus are for individual samples which were significantly different. Trophic levels are based on diets of the organism

$1442 \mathrm{ng} \mathrm{Cd}, 42 \%$ of the maximum observed in arctic cod, a remarkably high burden considering the body size of only $120 \mathrm{mg}$ dry weight ( $=12.0 \mu \mathrm{g} \mathrm{Cd} \mathrm{g}^{-1}$ ).

\section{DISCUSSION}

A primary abjective of this study was to collect organisms which represented a number of trophic levels and niches in the arctic marine system. In addition, it was important to collect adequate samples of arctic cod and Parathemisto libellula, 2 species which are dominant in the arctic marine community (Dunbar 1957, Bradstreet \& Cross 1982). The list of species summarized in Table 2 shows that a range of ecological groups was collected from both the epontic and the summer pelagic communities. A satisfactory range of body sizes was obtained for most organisms, thereby minimizing the effect of body size on the estimates of mean concentration and burden for each species.

\section{Comparison of groups within this study}

There was a low degree of variability between samples of the same organism taken at different times or places. This was particularly evident for the epontic community, chaetognaths, copepods, mysids, and to a lesser extent amphipods. Lack of significant withinspecies differences, but differences between most of the kinds of organisms, suggests that procedures in chemical analysis were satisfactory, with adequate control of contamination. Apparently our sampling methods avoided the usual major sources of metal contamination of plankton samples from sampling devices, hydrographic wire, or from rust, paint chips, and other sources on the research vessel (Martin \& Knauer 1973, Hamanaka \& Tsujita 1981)

Table 6. Comparison of means of pooled data (Table 5) using Scheffe's multiple range test. Means are in descending order of Cd concentration. Abbreviations along top of table correspond to species or group names listed in first column. Significant differences between means denoted by asterisk. Blank cells: no significant difference between species

\begin{tabular}{|c|c|c|c|c|c|c|c|c|c|c|c|c|c|c|c|c|c|}
\hline Taxa & Par & Hyp & Ga1 & Oni & Phy & $\mathrm{Ga} 2$ & Ano & Cop & Ep1 & Ep2 & Ar 1 & Cha & Cte & Boe & Ga3 & Ar2 & Mys \\
\hline Parathemisto libellula & - & & & & & $\cdot$ & - & • & $\cdot$ & $\cdot$ & · & $\cdot$ & - & $\cdot$ & $\cdot$ & - & · \\
\hline Hyperoche medusarum & & - & & & & $\cdot$ & $\cdot$ & $\cdot$ & $\cdot$ & • & $\cdot$ & $\cdot$ & $\cdot$ & $\cdot$ & $\cdot$ & $\cdot$ & $\cdot$ \\
\hline Gammarus setosus (Bay 12) & & & - & & & - & $\cdot$ & $\cdot$ & $\cdot$ & - & $\cdot$ & $\cdot$ & $\cdot$ & $\cdot$ & • & - & - \\
\hline Onisimus glacialis & & & & - & & & & · & $\cdot$ & $\cdot$ & $\cdot$ & $\cdot$ & $\cdot$ & $\cdot$ & • & $\cdot$ & $\cdot$ \\
\hline Phytoplankton (Cape Hatt) & & & & & - & & & & & & & & $\cdot$ & $\cdot$ & $\cdot$ & • & " \\
\hline Gammarus setosus (Kaujuitoq) & $\cdot$ & $\cdot$ & $\cdot$ & & & - & & & & & & $\cdot$ & $\cdot$ & $\cdot$ & $\cdot$ & $\cdot$ & $\cdot$ \\
\hline Anonyx sarsi & $\cdot$ & $\cdot$ & - & & & & - & & & & & & $\cdot$ & $\cdot$ & $\cdot$ & $\cdot$ & $\cdot$ \\
\hline Copeods & $\cdot$ & $\cdot$ & $\cdot$ & $\cdot$ & & & & - & & & & & $\cdot$ & $\cdot$ & $\cdot$ & $\cdot$ & $\cdot$ \\
\hline Epontic community $(<64 \mu \mathrm{m})$ & $\cdot$ & $\cdot$ & $\cdot$ & $\cdot$ & & & & & - & & & & & $\cdot$ & $\cdot$ & $\cdot$ & $\cdot$ \\
\hline Epontic community (> $64 \mu \mathrm{m}$ ) & $\cdot$ & $\cdot$ & $\cdot$ & $\cdot$ & & & & & & - & & & & & $\cdot$ & $\cdot$ & $\cdot$ \\
\hline Arctic cod (liver) & $\cdot$ & $\cdot$ & $\cdot$ & $\cdot$ & & & & & & & - & & & & & & $\cdot$ \\
\hline Chaetognaths & $\cdot$ & $\cdot$ & $\cdot$ & $\cdot$ & & $\cdot$ & & & & & & - & & & & $\cdot$ & $\cdot$ \\
\hline Ctenophores & $\cdot$ & $\cdot$ & $\cdot$ & $\cdot$ & $\cdot$ & $\cdot$ & - & • & & & & & - & & & & $\cdot$ \\
\hline Boeckismus edwarsdi & $\cdot$ & $\cdot$ & $\cdot$ & $\cdot$ & $\cdot$ & $\cdot$ & $\cdot$ & $\cdot$ & $\cdot$ & & & & & - & & & $\cdot$ \\
\hline Gammarus setosus (Z-lagoon) & $\cdot$ & $\cdot$ & $\cdot$ & $\cdot$ & $\cdot$ & $\cdot$ & $\cdot$ & $\cdot$ & $\cdot$ & $\cdot$ & & & & & - & & \\
\hline Arctic cod (whole body) & $\cdot$ & $\cdot$ & $\cdot$ & $\cdot$ & $\cdot$ & $\cdot$ & $\cdot$ & $\cdot$ & $\cdot$ & $\cdot$ & & ' & & & & - & \\
\hline Mysis litoralis & $\cdot$ & $\cdot$ & $\cdot$ & $\cdot$ & $\cdot$ & $\cdot$ & $\cdot$ & $\cdot$ & $\cdot$ & $\cdot$ & $\cdot$ & $\cdot$ & $\cdot$ & $\cdot$ & & & - \\
\hline
\end{tabular}


The only significant and substantial variation between samples of the same organism was for the intertidal amphipod Gammarus setosus. The cause is not clear but it does not seem to have been analytical or sampling error. The lack of significant difference between the 1982 and 1983 samples from Z-lagoon $\left(0.08\right.$ versus $0.07 \mu \mathrm{g} \mathrm{g}^{-1}$ wet weight), and the similarity between the gammarids and their food (i.e. epontic algae) indicated, first, a consistency in Cd concentration within the populations and, second, that gut contents may have influenced the $\mathrm{Cd}$ concentration of this species. Digestive tracts were not purged prior to analysis for any of the organisms, and the guts of gammarids collected under the ice in 1984 were full of algae. Also, the sandy substrate of Z-lagoon, where $G$. setosus had the lowest cadmium concentrations, was considerably different from either the rocky substrate of Bay 12 or the underside of the ice at Kaujuitoq. $G$. setosus is the only species in the present study which showed major variability; it appears to be real and may be related to gut contents.

The epontic community at Kaujuitoq did not differ appreciably in Cd content from the pelagic community at Cape Hatt. Samples of the under-ice community in both 1983 and 1984 contained approximately 1 to $2 \mu \mathrm{g}$ $\mathrm{g}^{-1}$ dry weight and were not significantly different from phytoplankton collected at Cape Hatt in 1983. The harpacticoid and calanoid copepods from the 2 communities also had similar $\mathrm{Cd}$ concentrations. Epontic Cd concentrations were similar in the $2 \mathrm{yr}$ although warm weather during the 1984 sampling season caused extensive runoff for a few days prior to sample collection. One possible reason for slightly elevated Cd concentrations in the surface waters of the Arctic Ocean is buildup of metal in snow as the result of atmospheric deposition over the winter, then release during spring melting, with similar inputs from melting glaciers and icebergs (Campbell \& Yeats 1982). If runoff water contained elevated $\mathrm{Cd}$ concentrations in 1984, there is no evidence that it influenced $\mathrm{Cd}$ content of the epontic community.

The $\mathrm{Cd}$ concentrations reported here are in reasonable agreement with the few published values from the Arctic (Table 5). In particular, Bohn \& McElroy (1976) report similar findings from Strathcona Sound on northern Baffin Island. Their concentrations of 2.8 and $5.0 \mu \mathrm{g}$ $\mathrm{g}^{-1}$ for copepods are slightly higher than the grand mean of $1.94( \pm \mathrm{SD}=1.40$ to 2.69$)$ given here, but their values of 1.2 to $1.3 \mu \mathrm{g} \mathrm{g}^{-1}$ for chaetognaths agree closely with the grand mean of $1.07( \pm \mathrm{SD}=0.72$ to 1.59) from the present study. Bohn \& McElroy reported whole-body cadmium of $0.62 \mu \mathrm{g} \mathrm{g}^{-1}$ for arctic cod from Strathcona Sound, close to the value of $0.40( \pm \mathrm{SD}=$ 0.21 to 0.76$) \mu \mathrm{g} \mathrm{g}^{-1}$ reported here for the same species from the same area.

\section{Comparison with regions outside the Arctic}

The concentration of dissolved $\mathrm{Cd}$ in arctic surface waters appears to lie consistently in the range of 0.03 to $0.06 \mu \mathrm{g} \mathrm{l}^{-1}$ (Moore 1981, Campbell \& Yeats 1982) which is marginally higher than the concentrations reported for other regions (Table 5). It has been hypothesized that adsorption of $\mathrm{Cd}$ by organic matter, the mechanism responsible for reducing the concentration of $\mathrm{Cd}$ to the low $n g ~^{-1}$ range in the surface waters of the Atlantic and the Pacific Oceans (Boyle et al. 1976, Bruland 1980, Boyle et al. 1981) is reduced in arctic waters because of the low quantity of dissolved organic material. Consequently, arctic surface waters $(<200 \mathrm{~m})$ tend to retain $\mathrm{Cd}$ which enters through fluvial inputs (Moore 1981, Campbell \& Yeats 1982) or from the melting of sea ice and glacier ice (Campbell \& Loring 1980, Campbell \& Yeats 1982) and do not demonstrate surface depletion relative to deeper waters (Moore 1981). Regardless of the mechanism involved, there is no indication that the marginally higher concentrations of dissolved Cd observed in surface waters of the Arctic Ocean are transferred to the biota examined in this study.

Mean $\mathrm{Cd}$ concentrations of all invertebrate organisms collected in this study were similar to, or less than, the concentrations in equivalent organisms outside of the Arctic (Table 5). This was particularly evident in the lower trophic levels, such as phytoplankton $(4.74 \mu \mathrm{g}$ $\left.\mathrm{g}^{-1}\right)$, the epontic community $\left(1.93 \mu \mathrm{g} \mathrm{g}^{-1}\right)$ and copepods $\left(1.94 \mu \mathrm{g} \mathrm{g}^{-1}\right)$ whose Cd concentration is generally a good indicator of the level of dissolved $\mathrm{Cd}$ in the water (Bruland et al. 1978, Davies 1978). Hamanaka \& Mishima (1981) report mean Cd concentrations of about $8 \mu \mathrm{g} \mathrm{g}^{-1}$ in copepods from the north Pacific Ocean, and 7 for the Bering Sea. The grand mean for the Canadian arctic copepods $\left(1.94 \mu \mathrm{g} \mathrm{g}^{-1}\right)$ is among the lower values reported for this group in the literature. Similarly, the arctic chaetognaths and mysids are not high in cadmium compared to those elsewhere.

Amphipods contained the highest concentrations of Cd but one group, the hyperiid amphipods, was consis. tently higher than any others. This is particularly noticeable in the Y-O-Y Parathemisto libellula collected at Cape Hatt, which were only 12 to $14 \mathrm{~mm}$ long but had 30 to $40 \mu \mathrm{g} \mathrm{Cd} \mathrm{g}^{-1}$. This is not a phenomenon of the Arctic, since equally high or higher $\mathrm{Cd}$ concentrations have been noted in hyperiid amphipods from the north Pacific Ocean and elsewhere by Hamanaka and co-workers (Table 5; Hamanaka \& Mishima 1981, Hamanaka \& Tsujita 1981, Hamanaka \& Ogi 1984). They have attributed the high levels to the carnivorous diet, and support for their hypothesis was given by the high Cd levels they found in copepods. 
In the present study, however, the Cd concentration in the food of hyperiids is as low as, or lower than that in food items of the omnivorous and herbivorous amphipods, yet the hyperiids are clearly higher in $\mathrm{Cd}$ concentration. Since diet (see below) apparently does not explain the high $\mathrm{Cd}$ in hyperiid amphipods, there must be some strong physiological affinity for the metal, such as adsorption onto the external surface or internal deposition, probably in association with metalbinding proteins. Whatever the mechanism, it is efficient, since Y-O-Y Parathemisto libellula had high concentrations in both June and August, and must have obtained it either by efficient transfer from the mother or by a rapid uptake in early spring.

Arctic cod had the second lowest average wholebody $\mathrm{Cd}$ concentration found in the study (Fig. 2), despite the fact that their diet is similar to that of the hyperiid amphipods. The selection of fish from other places for inclusion in Table 5 was guided by their documented importance in diet of northern seals, and in particular their availability to ringed seals from the Baltic Sea which have low Cd concentrations (Pertilla et al. 1983). The comparison with our fish data is not definitive because of differences in the tissues sampled (i.e. whole body versus muscle). The liver is a major site of accumulation, and it reached $1.14( \pm 1 \mathrm{SD}=0.77$ to 1.68) $\mu \mathrm{g} \mathrm{g}^{-1}$ in 6 livers of arctic cod collected at Grise Fiord. Bohn \& McElroy (1976) found somewhat less in livers from the eastern Canadian Arctic $\left[0.72 \mu \mathrm{g} \mathrm{g}^{-1}\right.$ ( $\pm 1 \mathrm{SD}=0.35$ to $1.09, n=15)$ ], but neither study gives evidence of accumulation to high levels in arctic cod. However, those 2 means are an order of magnitude higher than liver concentrations in cod from the Gulf of Bothnia (northern Baltic) $\left(0.06\right.$ to $0.18^{\dagger} \mu g^{-1}$; Pertilla et al. 1982). The benthic shorthorn sculpin Myoxocephalus scorpius from the Canadian Arctic (Strathcona Sound) is reported to have a mean value of $4.1 \pm$ $3.1 \mathrm{Mg} \mathrm{g}^{-1}(n=61)$ in liver (Bohn \& Fallis 1978). Muscle concentration in those sculpins was approximately $1.4 \mu \mathrm{g} \mathrm{g}^{-1}$, about 2 orders of magnitude higher than various measurements on muscle of Scandinavian cod and herring (Table 5). The Canadian arctic fish appear to have $\mathrm{Cd}$ values about 10 to 100 times higher than some fish species in the Baltic area. Among the organisms sampled in this study, only the arctic cod appeared to be high in $\mathrm{Cd}$ concentration compared to equivalent organisms elsewhere, even though they were very low compared to other arctic organisms. None of the epontic, phytoplankton or invertebrate samples were unusual in their $\mathrm{Cd}$ concentration. These levels in the biota do not support the concept of unusually high inputs of $\mathrm{Cd}$ to arctic marine ecosystems, either from pollution or natural causes. We reject the general hypothesis that higher $\mathrm{Cd}$ concentrations are characteristic of all arctic marine biota. The presence of high levels in marine mammals is acknowledged, as are the apparent smaller elevations in fish.

\section{Ecological significance of Parathemisto and arctic cod}

Both of these organisms are of great importance in arctic food chains because they are abundant and are consumed by most marine vertebrates. Thus they play a central role in the movement of energy from lower to upper trophic levels (Dunbar 1957, Bradstreet 1982).

The hyperiid amphipod Parathemisto libellula is most common in arctic waters (Dunbar 1957) although it is also in the western North Atlantic (Bowman 1960), and the north Pacific (Hamanaka \& Mishima 1981). It can reach a length of $6 \mathrm{~cm}$ over a life-span of 2 to $3 \mathrm{yr}$ (Bowman 1960). The diet of $P$. libellula is primarily calanoid copepods with smaller proportions of chaetognaths, fish larvae and other P. libellula (Dunbar 1946, Wing 1976). Smaller $P$. libellula feed mainly on smaller copepods, naupliae and phytoplankton (Wing 1976). $P$. libellula is not closely associated with the under-ice community and tends to remain in the water column, however it feeds on ice-associated copepods and possibly the epontic algae which slough off the ice (Bradstreet \& Cross 1982). The significance of $P$. libellula in arctic marine systems has been compared to that of the euphausiid Euphausia superba ('krill') in the Antarctic Ocean (Dunbar 1946, 1957). When arctic cod become scarce through population fluctuations (Bradstreet 1982) or during summer offshore movements, many arctic vertebrates feed on invertebrates, particularly $P$. libellula. Hyperiid amphipods may average $25 \%$ by weight of the diet of ringed seals (LGL 1983), and sometimes the gut contents of eastern Canadian arctic seals are almost exclusively invertebrates (Dunbar 1957, McLaren 1958). A young female ringed seal accidentally caught at Cape Hatt during the present study had gut contents consisting solely of hyperiid amphipods.

Arctic cod are small gadid fish (maximum size only 23 to $38 \mathrm{~cm}$ at age 5 to $6 \mathrm{yr}$ ). They are common throughout a circumpolar range and found either in the water column or near the bottom (Bohn \& McElroy 1976 , Lowry \& Frost 1981). Their diet is copepods, mysids, gammarid and hyperiid amphipods and some fish in spring and summer (Bradstreet \& Cross 1982). Youngof-the-year cod feed primarily on phytoplankton and copepod nauplii (LGL 1983). In turn, arctic cod are the basic component of the diets of virtually all other vertebrates in arctic marine systems. Frost \& Lowry (1981) report a total of 12 species of mammals, 20 birds, and 4 fishes which use arctic cod as a major portion of their diet in north Alaskan waters. Arctic cod average $65 \%$ 
(by weight) of the diet for all seabirds and $50 \%$ (by weight) for all marine mammals in eastern Lancaster Sound and western Baffin Bay (LGL 1983). A single harp seal contained the remnants of 541 arctic cod averaging $8 \mathrm{~cm}$ in length (Finley \& Gibb unpubl.).

From this comparison, it is evident that Parathemisto libellula and arctic cod have similar diets and play very similar roles within the arctic marine community. The extreme differences in their Cd concentration, however, would indicate considerable differences in the quantity of $\mathrm{Cd}$ transferred to the upper trophic levels, with considerably less $\mathrm{Cd}$ moving through cod than through the amphipod.

This difference is accentuated when the 2 species are compared on an energy content basis. Assuming that Parathemisto libellula and arctic cod contain 4.5 and $5.5 \mathrm{kcal} \mathrm{g}^{-1}$ dry weight respectively (estimates based on values cited in Lowry et al. 1980 and Bradstreet \& Cross 1982) then the 2 species would contain $2.55 \mu \mathrm{g}$ $\mathrm{Cd} \mathrm{kcal}^{-1}$ and $0.07 \mu \mathrm{g} \mathrm{Cd} \mathrm{kcal}{ }^{-1}$ respectively. Ringed seals feeding at $1600 \mathrm{kcal} \mathrm{d}^{-1}$ (Lowry et al. 1980) would

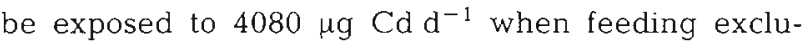

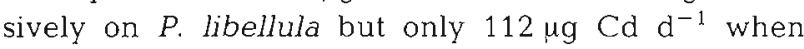
feeding only on arctic cod. By contrast humans are usually exposed to 35 to $40 \mu \mathrm{g} \mathrm{Cd} \mathrm{d} \mathrm{d}^{-1}$ through the diet (reviewed by Ryan et al. 1982). Consequently, a simple dietary influence, resulting from the unique arctic marine food web, may explain the high $\mathrm{Cd}$ levels in Canadian arctic seals, an hypothesis that could be tested by more precise modelling of these mammalian diets.

Acknowledgements. The authors gratefully acknowledge the assistance of the personnel of the BIOS camp at Cape Hatt, in particular Peter Blackall, Gary Sergy and Kelly McGregor and the LGL staff of William Cross, Mike Fabijan, Anne Maltby and Carole Martin for the use of equipment and assistance in identification of organisms. We also thank Jeff Anning and Glenn Cota, Bedford Institute of Oceanography, Dartmouth, Nova Scotia (1983), Dr H. Welch (1984), and the Polar Continental Shelf Project for the use of their sampling equipment and other facilities at Kaujuitoq and Glenn Williams of Avatuq Services, Arctic Bay for the collection of the arctic cod. C. R. M. personally thanks Neil Hutchinson and Don Lewis for their assistance in the field at Cape Hatt. Funding for this project was provided by a Strategic Grant (Oceans) from the National Science and Engineering Research Council of Canada, an NSERC Graduate Scholarship to C. R. M., and Northern Training Grants from the Association of Canadian Universities for Northern Studies.

\section{LITERATURE CITED}

Amiard-Triquet, C., Metayer, C., Amiard, J.-C. (1980). Etude du transfert de $\mathrm{Cd}, \mathrm{Pb}, \mathrm{Cu}$ et $\mathrm{Zn}$ dans les chaines trophiques neritiques et estuariennes - II. Accumulation biologique chez les poissons planctonophages. Wat. Res. 14: $1327-1332$
Bohn, A., Fallis, B. W. (1978). Metal concentrations (As, Cd, $\mathrm{Cu}_{1} \mathrm{~Pb}$ and $\mathrm{Zn}$ ) in shorthorn sculpins, Myoxocephalus scorpius (Linnaeus) and arctic char, Salvelinus alpinus (Linnaeus) from the vicinity of Strathcona Sound, Northwest Territories. Wat. Res. 12: 659-663

Bohn, A., McElroy, R. O. (1976). Trace metals (As, Cd, Cu, Fe and $\mathrm{Zn}$ ) in arctic cod, Boreogadus saida, and selected zooplankton from Strathcona Sound, northern Baffin Island. J. Fish. Res. Bd Can. 33: 2836-2840

Bowman, T. E. (1960). The pelagic amphipod genus Parathemisto (Hyperiidea: Hyperiidae) in the north Pacific and adjacent Arctic Ocean. Proc. U.S. natn. Mus. 112: 343-392

Boyle, E. A., Huestad, S. S., Jones, S. P. (1981). On the distribution of copper, nickel and cadmium in the surface waters of the north Atlantic and north Pacific Ocean. J. geophys. Res. 86: 8048-8066

Boyle, E. A., Sclater, F., Edmond, J. M. (1976). On the marine geochemistry of cadmium. Nature, Lond. 263: 42-44

Bradstreet, M. S. W. (1982). Occurrence, habitat use and behavior of seabirds, marine mammals and arctic cod at the Pond Inlet ice edge. Arctic 35: 28-40

Bradstreet, M. S. W., Cross, W. E. (1982). Trophic relationships at high Arctic ice edges. Arctic 35: 1-12

Brugmann, L. (1981). Heavy metals in the Baltic Sea. Mar Pollut. Bull. 12: 214-218

Bruland, K. W. (1980). Oceanographic distributions of cadmium, zinc, nickel, and copper in the north Pacific. Earth Planet. Sci. Lett. 37: 38-54

Bruland, K. W., Knauer, G. A., Martin, J. H. (1978). Cadmium in northeast Pacific waters. Limnol. Oceanogr. 23: 618-625

Campbell, J. A., Loring, D. H. (1980). Baseline levels of heavy metals in the waters and sediments of Baffin Bay. Mar. Pollut. Bull. 11: 257-261

Campbell, J. A., Yeats, P. A. (1982). The distribution of manganese, iron, nickel, copper and cadmium in the waters of Baffin Bay and the Canadian Arctic Archipelago. Oceanologica Acta 5: 161-168

Cherry, W. H. (1981). Distribution of cadmium in human tissues. In: Nriagu, J. (ed.) Cadmium in the environment. Part II. Health effects. John Wiley \& Sons, New York, p. $69-536$

Danielsson, L.-G., Magnusson, B., Westerlund, S. (1985). Cadmium, copper, iron, nickel, and zinc in the north-east Atlantic Ocean. Mar. Chem. 17: 23-41

Davies, A. G. (1978). Pollution studies with marine plankton. Part II. Heavy metals. Adv. mar. Biol. 15: 381-508

Dixon, R. (1981). Cadmium and its compounds: health impact of production and usage in the workplace. Standards and Programs Branch. Occupational Health and Safety Division, Ministry of Labour, Ontario

Dixon, W. J., Brown, M. B., Engleman, L., Frame, J. W., Hill, M. A., Jennrich, R. I., Toparek, J. D. (1983). BMDP statistical software. University of California Press, Berkeley

Dunbar, M. J. (1946). On Themisto libellula in Baffin Island coastal waters. J. Fish. Res. Bd Can. 6: 419-434

Dunbar, M. J. (1957). The determinants of production in northem seas: a study of the biology of Themisto libellula Mandt. 'Calanus' series. No. 14. Can. J. Zool. 35: 797-819

Eaton, A. (1976). Marine geochemistry of cadmium. Mar. Chem. 4: 141-154

Friberg, L., Piscator, M., Nordberg, G. F., Kjellstrom, T. (1974). Cadmium in the environment, 2nd edn. CRC Press, Cleveland, Ohio

Frost, K. J., Lowry, L. F. (1981). Trophic importance of some marine gadids in northern Alaska and their body-otolith size relationships. Fish. Bull. U.S. 79: 187-192

Goldblatt, C. J., Anthony, R. G. (1983). Heavy metals in 
northern fur seals (Callorhinus ursinus) from the Pribiloff Islands, Alaska. J. environ. Qual. 12: 478-482

Hamanaka, T., Mishima, S. (1981). Cadmium and zinc concentrations in marine organisms in the northern north Pacific Ocean. Res. Inst. N. Pac. Fish. Hokkaido Univ., Spec. Vol., p. 191-206

Hamanaka, T., Ogi، H. (1984). Cadmium and zinc concentrations in the hyperiid amphipod Parathemisto libellula from the Bering Sea. Bull. Fac. Fish. Hokkaido Univ. 35: $171-178$

Hamanaka, T., Tsujita, T. (1981). Cadmium and zinc concentrations in zooplankton in the subarctic region of the north Pacific. J. oceanogr. Sci. Japan 37: 160-172

Johansen, P., Kapel, F. O., Kraul, I. (1980). Heavy metals and organochlorines in marine mammals from Greenland. Coun. Meet. int. Coun. Explor. Sea C. M.-ICES/E: 32, p. 1-11

Knauer, G. A., Martin, J. H. (1973). Seasonal variations of cadmium, copper, manganese, lead and zinc in water and phytoplankton in Monterey Bay, California. Limnol. Oceanogr. 18: 597-604

Kremling, K. (1985). The distribution of cadmium, copper, nickel, manganese, and aluminium in surface waters of the open Atlantic and European shelf area. Deep Sea Res. 32: $531-555$

Lande, E. (1977). Heavy metal pollution in Trondheimsfjorden, Norway, and the recorded effects on the flora and fauna. Environ. Pollut. 12: 187-198

Leatherland, T. M., Burton, J. D., Culkin, F., McCartney, M. J., Morris, R. J. (1973). Concentrations of some trace metals in pelagic organisms and of mercury in northeast Atlantic Ocean water. Deep Sea Res. 20: 679--685

LGL (1983). Biological environment of eastern Lancaster Sound and western Baffin Bay: components and important processes. Environmental Studies Report No. 30. Department of Indian and Northern Affairs, Ottawa

Lowry, L. F., Frost, K. J. (1981). Distribution, growth, and foods of arctic cod (Boreogadus saida) in the Bering, Chukchi, and Beaufort Seas. Can. Fld Nat. 95: 186-191

Lowry, L. F., Frost, K. J., Burns, J. J. (1980). Variability in the diet of ringed seals, Phoca hispida, in Alaska. Can. J. Fish. aquat. Sci. 37: 2254-2261

Martin, J. H., Broenkow, W. W. (1975). Cadmium in plankton: elevated concentrations off Baja California. Science 190: 884-885

Martin, J. H., Bruland, K. W., Broenkow, W. W. (1976). Cadmium transport in the California current. In: Windom, $\mathrm{H}$. L., Duce, R. A. (eds.) Marine pollutant transfer. D. C. Heath \& Co., Lexington, Mass., p. 159-184
Martin, J. H., Knauer, G. A. (1973). The elemental composition of plankton. Geochim. Cosmochim. Acta 37: 1639-1653

McLaren, I. A. (1958). The biology of the ringed seal (Phoca hispida Schreber) in the eastern Canadian Arctic. Fish. Res. Bd Can. Bull. 118: 1-97

Miettinen, V., Verta, M. (1978). On the heavy metals and chlorinated hydrocarbons in the Gulf of Bothnia in Finland. Finnish mar. Res. 244: 219-226

Moore, R. M. (1981). Oceanographic distributions of zinc, cadmium, copper and aluminium in waters in the central Arctic. Geochim. Cosmochim. Acta 45: 2475-2482

Pertilla, M., Stenman, O., Pyysalo, H., Wickstrom, K. (1986). Heavy metals and organochlorine compounds in seals in the Gulf of Finland. Mar. environ. Res. 18: 43-59

Pertilla, M., Tervo, V., Parmanne, R. (1982). Heavy metals in Baltic herring and cod. Mar. Pollut. Bull. 13 (11): 391393

Polikarpov, G. G., Oregioni, B., Parchevskaya, D. S., Benayoun, G. (1979). Body burden of chromium, copper, cadmium and lead in the neustonic copepod Anomalocera patersoni (Pontellidae) collected from the Mediterranean Sea. Mar. Biol. 53: 79-82

Rahn, K. A. (1981). Atmospheric, riverine and oceanic sources of seven trace constituents to the Arctic Ocean. Atmos. Environ. 15: 1507-1516

Ryan, J. A., Pahren, H. R., Lucas, J. B. (1982). Controlling cadmium in the human food chain: a review and rationale based on health effects. Environ. Res. 28: 251-302

Schafer, H. A., Herschelman, G. P., Young, D. R., Mearns, A. J. (1982). Contaminants in ocean food webs. In: Bascom, W. (ed.) Coastal water research project. Biennial report for the years 1981-1982. South California Coastal Water Research Project, Long Beach, Calif., p. 17-28

Szefer, P., Skwarzec, B., Koszteyn, J. (1985). The occurrence of some metals in mesozooplankton taken from the southern Baltic. Mar. Chem. 17: 237-253

Thomas, D. J., Macdonald, R. W., Cornford, A. B. (1983) Chemical and biological studies at Strathcona Sound N. W. T. 1982. Final report. Department of Indian Affairs and Northern Development, Northern Affairs Program, N. W. T. Region, Canada

Wagemann, R., Snow, N. B., Lutz, A., Scott, D. P. (1983) Heavy metals in tissues and organs of the narwhal (Monodon monoceros). Can. J. Fish. Aquat. Sci. 40 (Suppl. 2): $206-214$

Wing, B. L. (1976). Ecology of Parathemisto libellula and $P$. pacifica (Amphipoda: Hyperiidae) in Alaskan coastal waters. Ph.D. thesis, University of Rhode Island, Kingston 